\title{
Design and Implementation of Integrated Smart Township
}

\author{
Shashank Singh ${ }^{1}$, Tarun Sharma ${ }^{2}$, Pankaj Bande ${ }^{3}$
}

\begin{abstract}
The objective is to develop a Smart-Township which can be controlled from all over the world wirelessly. Smart-Township is a group of stationary smart homes which provides distributed sensing, computation and communication in the environment. To integrate information from individual smart-home and to transmit data to other smart-homes we have divided them in to sectors, it will provide us a better way to analyze each home, sector as well as whole township easily. We further did some development in proposed township in such a way that every home is able to communicate with other home of the township, we outline implementation of smart-home into a smart township.
\end{abstract}

Keywords: PIC microcontroller, C-18 cross compiler, MP-LAB software, RF circuit, DTMF.

\section{Introduction}

The Smart homes of the future promises to change the way we live, wirelessly connected and operated appliances, efficient use of energy are some of the features of future homes and towns. The smart township presented in this paper uses cutting edge technology to enhance the smartness of a town. Here the township is divided into four sectors, each sector has several houses controlled and connected wirelessly. The heart of the whole township is an intelligent module having a PIC18F4550 micro-controller and various peripherals such as LCD display, relays to operate AC appliances, LEDs, Buzzer etc. The intelligent part of the whole township is its accessibility to be controlled wirelessly with GSM technology from anywhere in the world.

Every smart-home is equipped with an intelligent module containing PIC microcontroller which was programmed by MP-Lab software in Embedded-C language, in such a way that each home communicates with each other and even can send information wirelessly. A home can send invitation wirelessly to others (e.g. birthday etc.). This township has got the ability to control individual home appliances (230V A.C), a particular sector or complete township by RFID, Phone call, Computer and Internet (e.g. Skype ${ }^{\mathrm{TM}}$, Team Viewer ${ }^{\mathrm{TM}}$ etc.).

Definitely this concept is not adoptable in developing countries like India and others but give it can pave path for the development of smart homes in future. At present mobile phones are becoming an integral part of human life, so people will be curious to use this concept as its application is user friendly.

Proposed system has been tested practically and gives expected results.

\section{System architecture}

The architecture of the whole township can be broken down in two parts, the transmitting end and the receiving end as shown in Figure 1.1 \& Figure 1.2. The DTMF IC CM8870 and HT12E are the basic components of the transmitting side, while the receiving end is composed of receiver circuit connected to microcontroller along with its peripherals.

\section{A. Transmitter End-}

a. CM8870- It provides full DTMF receiver capability. A CMOS Integrated DTMF receiver which uses digital counting techniques for the detection and decoding of all 16 DTMF tone pairs into a 4-bit binary code.

b. HT12E- The $2^{12}$ series of encoder pings 4 -word transmission during transmission enable. This process remains continue till transmission enable goes low. The $2^{12}$ decoders are a series of CMOS LSIs for remote control system applications.

c. Cell phone or Computer- These devices are for generation of DTMF tones. These peripherals act as input to DTMF receiver.

\section{B. Receiver End-}

a. PIC18F4550- It is 16 bit MCU available in 40-pin DIP packing, having 100,000 erase/write cycle enhanced flash program memory along with 1,000,000 erase/write cycle data EEPROM Memory. Data is retained in this controller till 40 years without refreshing.

b. HT12D- The $2^{12}$ series of decoders are capable of decoding information's that consist of $\mathrm{N}$ bits of address and $12-\mathrm{N}$ bits of data. HT12D is arranged to provide8 address bits and 4 data bits. The $2^{12}$ decoders are a series of CMOS LSIs for remote control system applications. Its 4 output pins are connected to controller's port pins.

c. Relays- Our system comprises with 3 SPDT relays. These relays act on 230V A.C supply. Each relay embeds with one A.C appliance. Controller output makes relay on and off. 
d. LM35-The LM35 series ICs are precision temperature sensors, whose output voltage is linearly proportional to the Celsius (Centigrade) temperature. This detector gives us sensing of temperature rise which is necessary to the safety point of view. During temperature rise it sends input to controller. Controller displays temperature rise on LCD and warn by buzzer beeping.

e. LCD- Liquid Crystal Display (LCD) is also an integral part of receiver end. Whatever operation is being carried on is displayed on LCD. It gives humans a medium to communicate with electronics. We have used 16x2 Alphanumeric LCD.

f. Buzzer \& LEDs- In order to bring working of electronics circuit in range of audible and visible range we used buzzer and LEDs. LED blinking indicates data transmission. Buzzer beeping is also for same job by sound.

(Two computers connected via internet)

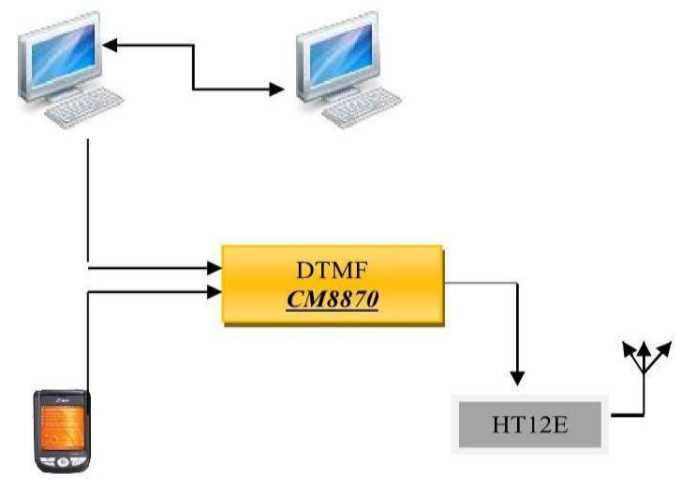

Figure 1.1 Transmitting End

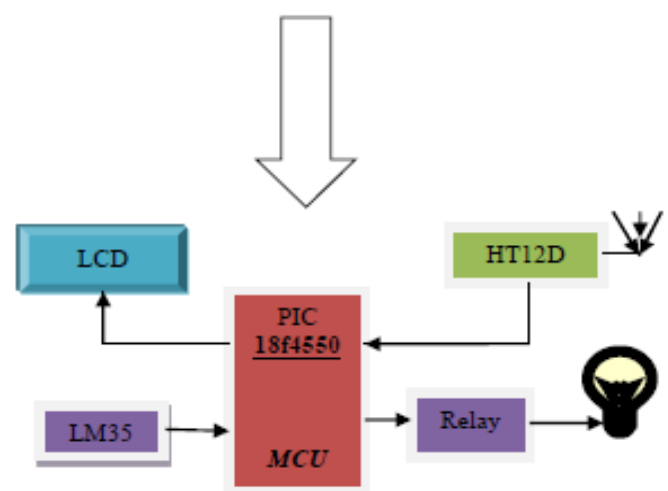

Figure 1.2 Receiving End (Microcontroller communicating with its periphery)

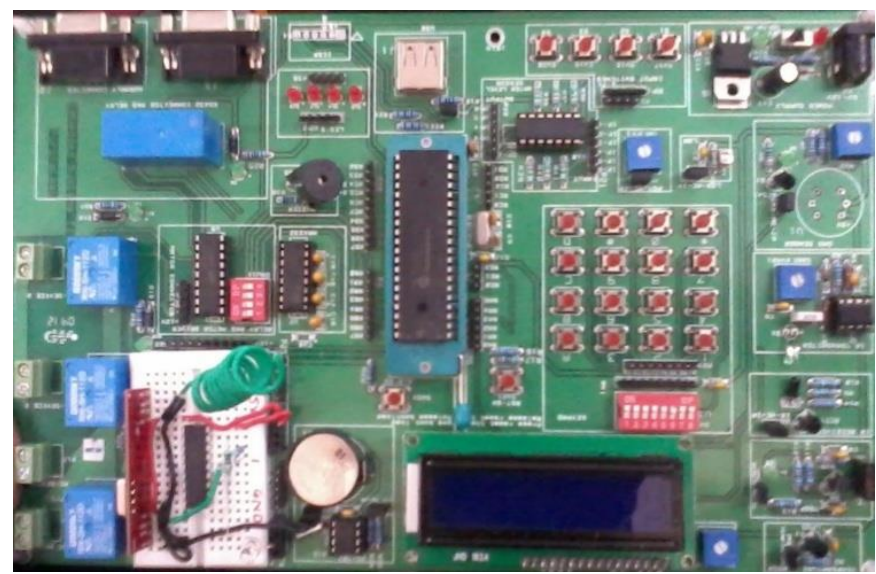

Figure 1.3 Receiver Board

\section{Township control}

The Intelligent township control increases comfort, energy saving, security and pleasant lifestyle in a township. Here we have employed distributed control system that is a computerized, intelligent network of electronic devices to monitor and control homes, sectors of township. 


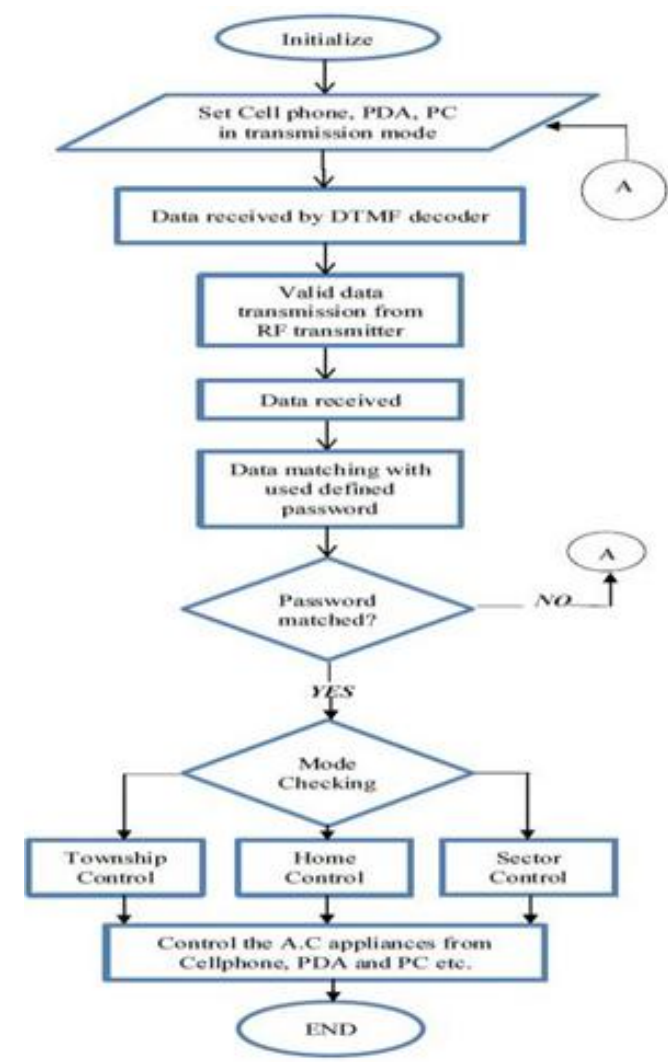

Figure 1.4 Flow chart of Township control

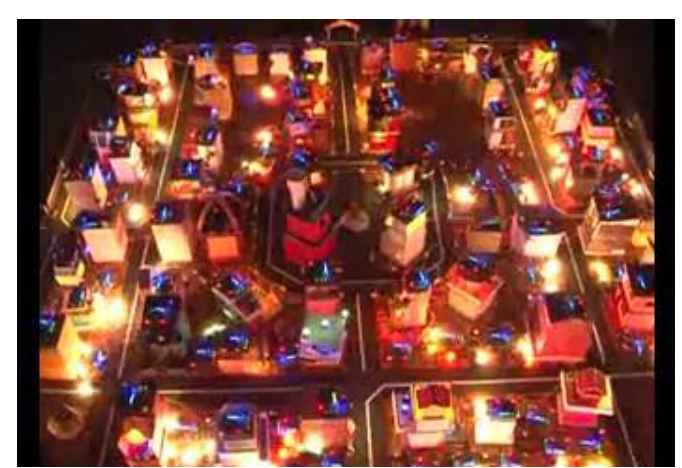

Figure 1.5 Actual Township Footage

Smart Township has ability to communicate within a restricted range, have a fixed location, and have a unique integer identifier (ID number). One can get access to these by cracking password i.e. unique id.

Whole Township has one integer identifier. Each smart home has unique integer identifier. Every sector also has unique ID number. User can get access to respective one by cracking unique password only. Microcontroller is the key device in our township. It interacts with input and output devices and communicates with user through different terminal device. Input device to controller are temperature sensor and RF receiver.

1. Power on the supply of all devices.

2. User sends data from input device.

3. Data received by controller through $433 \mathrm{MHz}$ radio frequency.

4. Controller embedded with a 'C' program.

5. Program describes about defined password e.g. Township control, Home control and Sector control.

6. As parameter defined by user respective control attained by user.

7. Once control gets into user hand then one can remotely control Entire Township, individual home and one sector.

\section{SECTOR CONTROL}

Our township has four sectors, each sector is a set of some smart home, and presence of sector in township makes it well ordered. With sector availability it is easy to assign location of each home in township. 
So we can say our sector as smart sector because likewise every home every sector has got the ability of get controlled wirelessly. We can access each sector individually. This feature makes township energy efficient. Suppose if any fault occurred in any of sector then we can isolate that sector from township without interrupting supply of complete township.

Also due to presence of smart sectors it becomes easy for robotic taxies to find the location of every home. Robotic taxies are autonomous robots which are capable to reach location of any home within township.
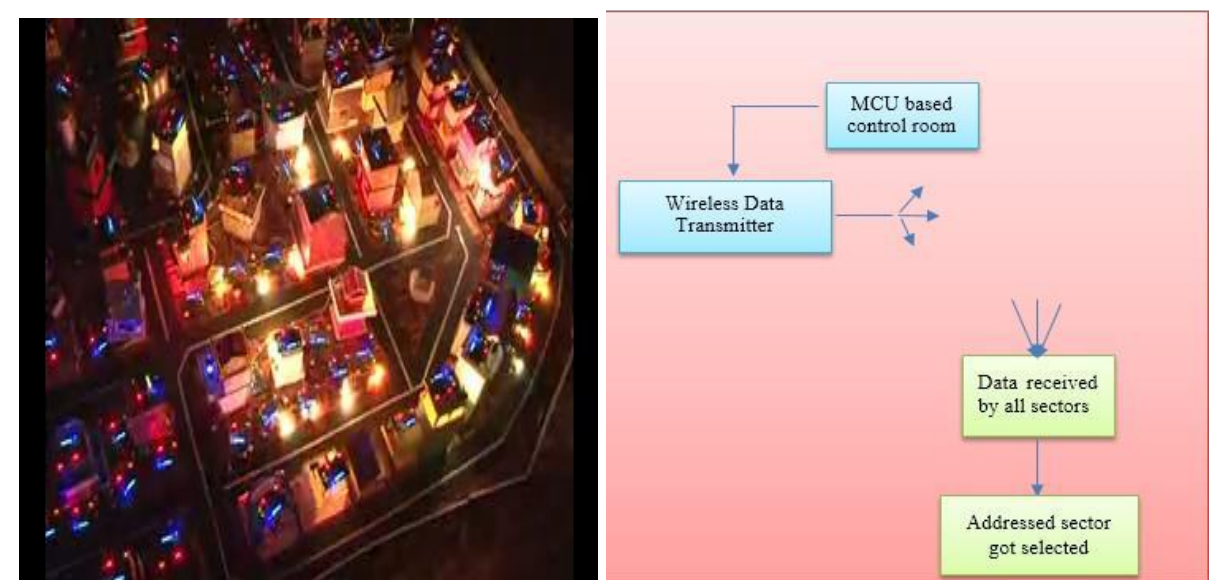

Figure 1.6 Actual Sector Footage

Figure 1.7 Structural Diagram for sector

\section{Features}

We have called our township as Smart Township. We have called it so because it has not only taken care of implementation of technology in home but also focused on basic need of humans. In a township basic essentials are water supply, transportation, fire station, hospital, electric supply, proper communication among people etc.

\section{A. Water Tank-}

Water is one of the essential requirements of human life. Human life on earth without water is impossible. Township got an integrated water tank which contained water level indicator. Water level indicator basically indicates level of water in water tank. Level of water is determined in five stages.
a. Water Tank Full.
b. Level $75 \%$.
c. Level $50 \%$.
d. Level $25 \%$.
e. Water Tank empty.

Output of water level indicator can be one among five. Water level indicator is connected to RF transmitter. Through RF transmitter lever of water is send to township. Every home has LCD display. Level of water is displayed on that.

This feature enhances the smartness of our cluster.

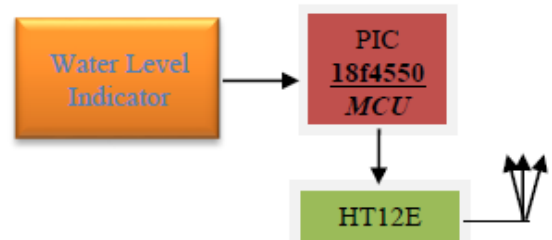

Figure 1.8 Transmitter of Water Level Indicator

\section{B. Robotic Taxies-}

Public Transport is another essential requirement of society. Every stationary element of our cluster has fixed location. Autonomous robotic taxies are capable to reach location of stationary element of cluster. 


\section{Gates \& Road Lights-}

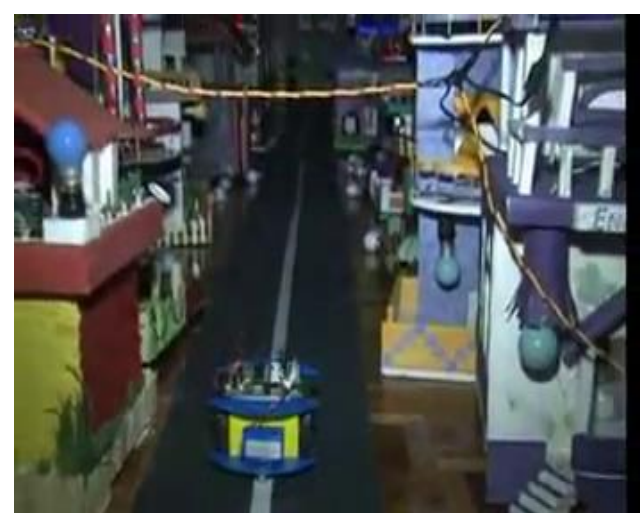

Figure 1.9 Robotic Taxi

Township has RF controlled gates. Township got a control room. From control room we can control gates. Gates combines with servo motor. Servo motor has 180 degrees' rotation. This ability makes servo motors perfect for making gate of township. There are roads within township for autonomous robotic taxies. Every road has road lights which are controlled wirelessly.

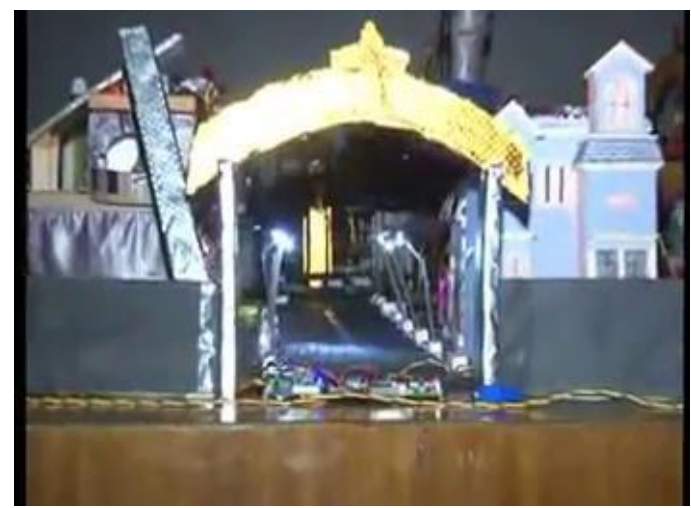

Figure 1.10 RF Controlled Gate

Wireless control of road lights makes easily switch off light during day time. This makes our township energy efficient.

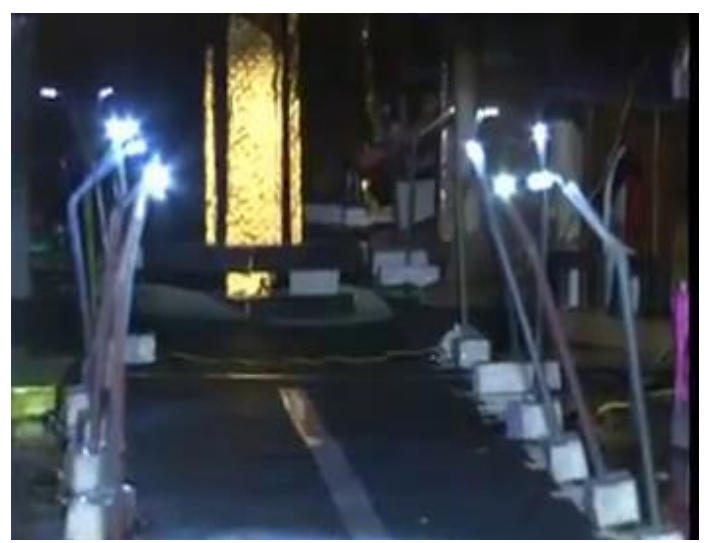

Figure 1.11 Road Lights

\section{Individual Smart Home-}

Stationary elements of our cluster have also intelligence. Each element consists of receiver board (Figure 1.3). One element can embed with transmitter circuit within individual home receiver board. This will ensure communication among every home also. This brings intelligence to receiver end. 


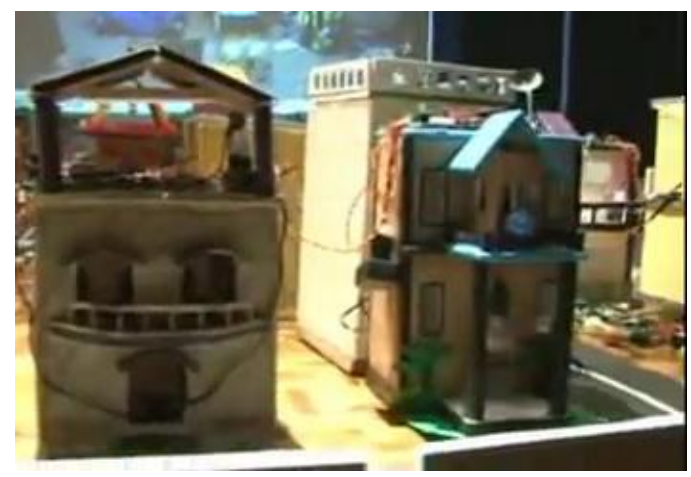

Figure 1.12 Individual Smart-home

Due to lack of budget we have only put intelligence to transmitter side only. That is why each board inside individual home has only capability of receiving data.

\begin{tabular}{|l|l|}
\hline \multicolumn{1}{|c|}{ Communication } & \multicolumn{1}{|c|}{ Frequencies } \\
\hline RF Tx-Rx & $433 \mathrm{MHz}$ \\
\hline GSM & $900 \mathrm{MHz}$ \\
\hline DTMF tone & $697-1477 \mathrm{~Hz}$ \\
\hline Buzzer & $20 \mathrm{~Hz}-20 \mathrm{KHz}$ \\
\hline LEDs \& LCD & $430-790 \mathrm{THz}$ \\
\hline Wi-Fi & $2.4 \mathrm{GHz}$ \\
\hline
\end{tabular}

Table 1 Frequencies allocation in Township

\section{Conclusion}

We have developed an improved township model, set of several smart-homes, that allows for dynamic updating of clusters. An implementation of this protocol for embedded systems has also been developed.

The presented township model in this paper is realized practically, tested for multi modes of operation and gave an excellent control of the home appliances under test. The system presented in this paper introduced a friendly system to control the home appliances remotely by the use of mobile cell phones, PDA, LAPTOP or PC.

The proposed system is characterized by its grand features required for the modern home automation system such as flexibility, security, friendly, in addition to the existence of feedback on line messages to inform the master about the state of the system and the appliances.

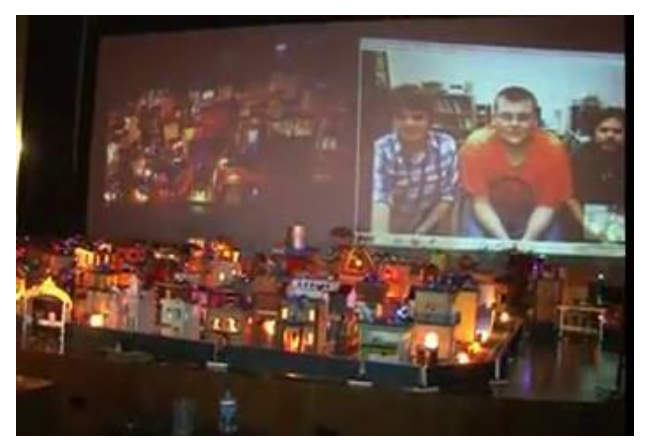

Figure 1.13 Controlling Township Live from Oklahoma State University, USA via Skype ${ }^{\mathrm{TM}}$

\section{Acknowledgements}

This project work is supported by Babu Banarasi Das Group of Educational Institution, Lucknow and Purple Leap (A Educomp-Pearson Company), Bangalore, India.

Authors can be contacted by shashanksingh0110@gmail.com ${ }^{1}$, sharmatarunsarma7@gmail.com², pankaj.bande@wipro.com ${ }^{3}$.

\section{References}

[1]. Gregory J. Barlow, Thomas C. Henderson, Andrew L. Nelson, and Edward Grant. "Dynamic Leadership Protocol for Snets." Proceedings of the IEEE International Conference on Robotics and Automation. New Orleans, LA. April 2004. pp. 10911096. 
[2]. M. s. Nasr and F. H. A. s. Azwai, "Friendly home automation system using cell phone and J2ME with feedback instant voice messages," Computer Systems and Applications, 2009. AICCSA 2009. IEEE/ACS International Conference on, Rabat, 2009, pp. 531-538.

[3]. H. Huang, S. Xiao, X. Meng and Y. Xiong, "A Remote Home Security System Based on Wireless Sensor Network and GSM Technology," Networks Security Wireless Communications and Trusted Computing (NSWCTC), 2010 Second International Conference on, Wuhan, Hubei, 2010, pp. 535-538.

[4]. Zhenxing Wang, Zhongyuan Liu and Linxiang Shi, "The smart home controller based on zigbee," Mechanical and Electronics Engineering (ICMEE), 2010 2nd International Conference on, Kyoto, 2010, pp. V2-300-V2-302. 